\title{
Investigation of soliton bound states in the Raman spectrum of pure and doped spin-Peierls chains
}

\author{
D. Augier ${ }^{a}$, E. Sørensen ${ }^{a}$, J. Riera ${ }^{a b}$ and D. Poilblanc ${ }^{a}$ \\ ${ }^{a}$ Laboratoire de Physique Quantique 6 Unité Mixte de Recherche 5626, Université P. Sabatier, 31062 Toulouse, France \\ ${ }^{b}$ Instituto de Fúsica Rosario, Consejo Nacional de Investigaciones Científicas y Técnicas y Departamento de Fúsica, \\ Universidad Nacional de Rosario, Avenida Pellegrini 250, 2000-Rosario, Argentina
}

(October 9, 2018)

\begin{abstract}
We investigate the occurrence of singlet bound states in the Raman spectrum of dimerized spin $1 / 2$ chains by Exact Diagonalization and Density Matrix Renormalization Group techniques. We predict that several bulk $\delta$-peaks could be observed in pure systems. Furthermore, we show that new low energy lines arise from non-magnetic impurity doping. These features are interpreted in terms of soliton-antisoliton and soliton-impurity bound states respectively. Energies and spectral weights associated with these bound states are sensitive to lattice relaxation effects. Our results are discussed in the context of the inorganic spin-Peierls compound $\mathrm{CuGeO}_{3}$ and quantitatively compared to recent Raman experiments.
\end{abstract}

\section{INTRODUCTION}

One-dimensional quantum spin chains have drawn great attention since the discovery of a spip Peierls transition in the inorganic $\mathrm{CuGeO}_{3}$ compoundt. The transition is experimentally inferred from an isotropic drop of the magnetic susceptibility below a critical temperature signaling the opening of a singlet-triplet spin gap $\Delta^{01}$ together with a lattice dimerization.

The occurrence of well-defined magnetic bound states below the excitation continuum has been intensively investigated both theoretically and experimentally. A triplet bound state with $\Delta^{01} \simeq 16.8 \mathrm{~cm}^{-1}$ has been observed by inelastic neutron scattering experiments Raman scattering experiments have identified a sharp singlet resonance at an energy $\Delta^{00} \simeq 29.7 \mathrm{~cm}^{-1}$ above the ground state 4 . Recently, Raman experiments on $\mathrm{Zn}$ doped $\mathrm{CuGeO}_{3}$ have detected a new singlet bound state at $\Delta_{1}^{\text {imp }} \simeq 15.0 \mathrm{~cm}^{-1}$ which is close to the value of the spin gap $\Delta^{01}$ (Ref. 8). Although it is commonly believed that these features signal the presence of bound states, there is still some controversy about their interpretation. It has been proposed that the singlet bound state is a magnon-magnon bound stated. An alternative description of both the singlet and triplet bound states, in terms soliton-antisoliton $(s \bar{s})$ bound states, is also possible 9, 10 . However, the question remains whether such $s \bar{s}$ bound states are observable in Raman spectroscopy. It is the purpose of the present paper to show that this is indeed the case: states which are clearly identifiable as $s \bar{s}$ or impurity-soliton bound states yield a significant Raman spectral weight at frequencies roughly coincident with the experimental results. Hence, a complete understanding of the experimental results is possible within the soliton picture both in pure and doped materials.

Quasi one-dimensional spin-Peierls compounds are widely described in the literature as frustrated dimerized Heisenberg chains 11 12:

$$
H=\sum_{i}\left[J\left(1+\delta(-1)^{i}\right) \vec{S}_{i} \cdot \vec{S}_{i+1}+J_{2} \vec{S}_{i} \cdot \vec{S}_{i+2}\right],
$$

where the explicit dimerization $\delta$ arises from some coupling to the three-dimensional lattice treated at the mean-field level. An extension of this model allowing for (slow) variation of the order parameter $\delta$ in space will be discussed latter on. We first summarize here some well known properties of the dimerized Heisenberg model (in the following we set $J=1$ ).

Systems with $\delta=0$. A spin gap smoothly opens at $J_{2 c} \simeq 0.2412$ (Ref. 13,14,12,15) accompanied by a spontaneous lattice dimerization. The ground state is then twofold degenerate. The Majumdar-Ghosh (MG) point 16 $\left(J_{2}=0.5\right.$ and $\left.\delta=0\right)$ is believed to correctly describe the physics when $J_{2 c}<J_{2} \leq 1 / 2$. The elementary excitations are depicted as solitons $(s)$ and antisolitons $(\bar{s})$ 17, topological defects between the two ground state patterns. No low energy $s \bar{s}$ bound states occur in this mode 10 and a continuum of magnetic excitations begins exactly at $\Delta^{00}=\Delta^{01}=2 \Delta_{\text {sol }}$ where $\Delta_{\text {sol }}$ is the minimal energy to create a soliton.

Systems with $\delta \neq 0$. For $J_{2}>J_{2 c}$, a non-zero $\delta$ lifts the degeneracy between the two ground states and creates a linear potential that confines solitons and antisolitons into bound states 9 . A ladder of $s \bar{s}$ bound states occurs below the continuum which begins at $2 \Delta^{01}$ r.e. when a higher energy $s \bar{s}$ pair decays into two pairs 10 (see Fig. 1). The number of $s \bar{s}$ bound states has to be determined numerically and appears to increase as the dimerization is decreased 18 . Turning on a small $\delta$ with $J_{2}<J_{2 c}$ creates a quite different situation. The explicit dimerization opens a spin gap $\Delta^{01} \propto \delta^{2 / 3}$ transforming the massless spinons of the Heisenberg chains into massive solitons. A mapping to the massive Thirring model gives evidences 
for the existence of exactly one triplet and one singlet bound states with a universal ratio $\Delta^{00} / \Delta^{01}=\sqrt{3}$ (Ref. $13,19,9$ ).

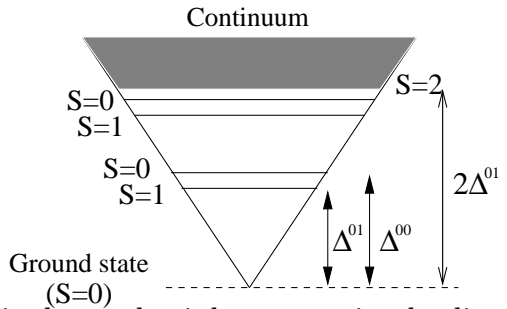

FIG. 1. Singlet and triplet states in the linear potential. The continuum begins with a $\mathrm{S}=2$ state (in finite systems).

In this paper, the Raman spectrum of dimerized Heisenberg chains is numerically investigated by Exact Diagonalization (ED) and Density Matrix Renormalization Group (DMRG) calculations. In section III, we discuss the possibility of observing $s \bar{s}$ singlet bound states using Raman spectroscopy on pure systems. In section III, we consider the effects of non-magnetic impurity doping on the Raman spectrum. Lattice relaxation is studied in section IV. Results of sections II, III and IV are obtained for parameters close to the MG point, useful to gain insight for a qualitative understanding. Finally, the relevance of these results to $\mathrm{CuGeO}_{3}$ are quantitatively discussed in section $\mathrm{V}$ in relation with recent experimental results.

\section{PURE COMPOUND}

Within the Loudon-Fleury theory20, the Raman operator is written as 61 .

$$
H_{R}=\sum_{i}\left[\vec{S}_{i} \cdot \vec{S}_{i+1}+\gamma \vec{S}_{i} \cdot \vec{S}_{i+2}\right] .
$$

Here, $\gamma$ is a microscopic parameter, and terms proportional to the Hamiltonian have been omitted. The Raman intensity $I_{R}$ can then be obtained:

$$
I_{R}(\omega)=\sum_{n}\left|\left\langle\Psi_{0}\left|H_{R}\right| \Psi_{n}\right\rangle\right|^{2} \delta_{\varepsilon}\left(\omega-E_{n}+E_{0}\right),
$$

where $\left|\Psi_{n}\right\rangle$ is a complete set of eigenstates with energy $E_{n}(n=0$ corresponds to the ground state $)$ and $\delta_{\varepsilon}$ is the lorentzian of width $\varepsilon$. The Raman operator $H_{R}$ depends on the value of $\gamma$. However, varying $\gamma$ only makes minute changes for the quantities of interest at least for the parameter values used in this paper. Hence, we shall use the operator $H_{R}=\sum_{i} \vec{S}_{i} \cdot \vec{S}_{i+1}$ in the following.

A typical Raman spectrum obtained by ED on a closed ring of 28 sites is shown in Fig. 2 a for $J_{2}=0.5, \delta=$ 0.05. These parameters are chosen for two reasons: $i$ ) to minimize finite-size effects, ii) to study the spectral weight of the two (possibly three) $s \bar{s}$ bound states that numerically have been identified for these parameters 10 . As can be seen in Fig. 2a, the Raman intensity is zero up to $\Delta^{00}$, where the first bulk bound state (BBS) occurs. This one is by far the most pronounced feature in the spectrum. The second bound state also has a significant weight while the third one is very weak and difficult to distinguish from the continuum without a precise analysis of finite-size effects.

The relative weights of the peaks are plotted in Fig. $2 \mathrm{~b}$ for lattice sizes from 16 to 28 sites. Note that the slightly larger dimerization $\delta=0.1$ used here reduces finite size effects but the number of bound states is lowered from three to two. At large lattice sizes, the weight of the two first peaks is constant and clearly non-zero signalling their bound state nature in contrast to the third peak which vanishes in the thermodynamic limit indicating that it is part of the continuum. An equivalent analysis for parameters closer to experimental values $\left(J_{2} \sim 0.36, \delta \sim 0.014\right)$ (Ref. 11) is difficult due to large finite size effects. However, the physics should be similar and it therefore appears reasonable to interpret the experimentally observed sharp peaks in pure systems as singlet $s \bar{s}$ bound states.

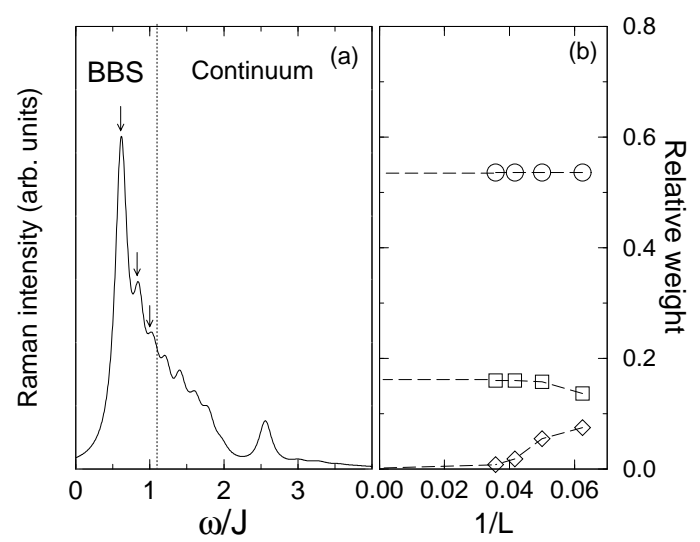

FIG. 2. (a) Raman intensity calculated for a $\mathrm{L}=28$ site ring with $J_{2}=0.5, \delta=0.05$ and a broadening $\varepsilon=0.1 J$. The positions of the three singlet bulk bound state (BBS) from Ref. 10 are indicated by arrows. The dashed line shows the onset of the continuum. (b) Relative weight of the three lowest energy peaks in the Raman spectrum for $J_{2}=0.5$ and $\delta=0.1$ as a function of the inverse lattice size. The presence of two $\operatorname{BBS}(\circ, \square)$ below the continuum $(\diamond)$ can then be inferred.

\section{DOPED COMPOUND}

We now study the effect of introducing in-chain nonmagnetic impurities. $\mathrm{CuGeO}_{3}$ can be doped with low concentrations of $\mathrm{Zn}$. The spin $0 \mathrm{Zn}$ substitutes the magnetic $\mathrm{Cu}^{2+}$ and in a first approximation the effect of such an impurity is simply to break the linear chains. Hence, 
we can model the effect of $\mathrm{Zn}$ doping by studying chains of different lengths with open boundary conditions.

First we qualitatively analyse the case of odd length chains. One end of the chain begins with a weak link $J(1-\delta)$. The chain end will now create a linear potential capable of binding a soliton. The ground state is made of a soliten bound to the chain end by this linear potential22, 23. 10.6. Other higher lying bound states in the linear potential may also been identified and we shall refer to these states as edge bound states (EBS). These EBS exist up to an energy $\Delta^{01}$ above the ground state, where it becomes energetically more favorable to create a free $s \bar{s}$ pair. As the invariance translation is broken, a pseudo-continuum due to the $s \bar{s}$ dispersion begins there. The lowest lying state of the pseudo-continuum should consequently be made of the solitonic GS plus a $s \bar{s}$ pair and have a spin 3/2 (the impurity bound soliton and the $s \bar{s}$ pair do not interact in the thermodynamic limit). A two-particle continuum can be observed when two $s \bar{s}$ pairs can be created, i.e. for energies larger than $2 \Delta^{01}$.

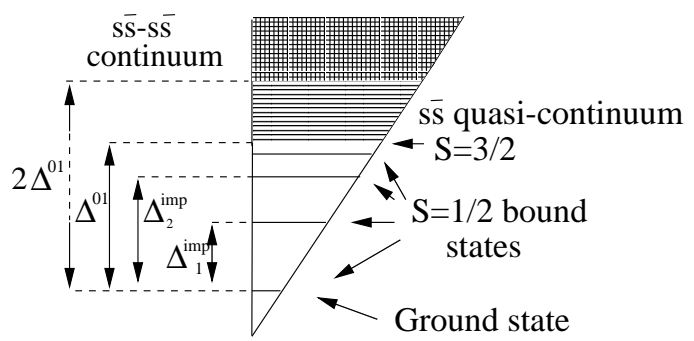

FIG. 3. The potential well created by an open boundary. The four soliton-impurity bound states shown here correspond to the parameters of Fig. $456\left(J_{2}=0.5, \delta=0.05\right)$.

We now turn to a more quantitative study. The number of EBS depends sensitively on the parameters of the Hamiltonian and previous parameters such as $J_{2}=0.5$ and $\delta=0.05$ should be optimal to observe EBS, with small finite size effects. Fig. 1 shows infinite size extrapolations of the energies obtained with ED or a DMRG treatment for these parameter values. Four soliton-impurity bound states below the continuum can be clearly identified. As discussed before, the energy of the soliton-impurity bound state has a finite upper limit and the onset of the pseudo-continuum is indicated by a spin $3 / 2$ state. We have checked that its energy with respect to the GS is, in the thermodynamic limit, the lowest energy of a $s \bar{s}$ bound state, i.e. the spin gap $\Delta^{01}=\left(0.5200 \pm 10^{-4}\right) J$. More explicitly, one finds (see Fig. 3 for the notation) $\Delta_{1}^{\mathrm{imp}} \simeq 0.20 \mathrm{~J}, \Delta_{2}^{\mathrm{imp}} \simeq 0.35 \mathrm{~J}$ and $\Delta_{3}^{\mathrm{imp}} \simeq 0.47 \mathrm{~J}$.

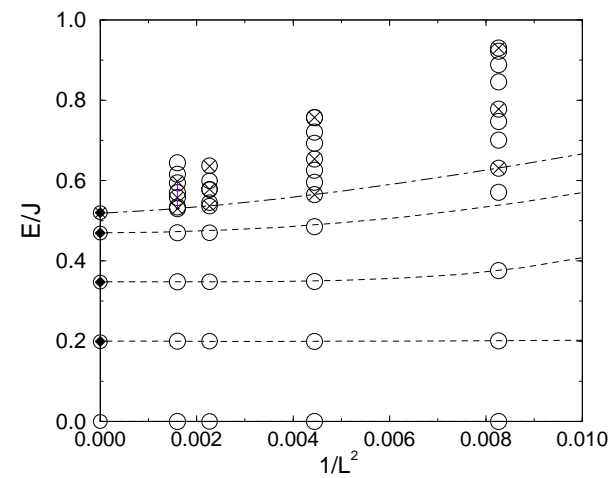

FIG. 4. Lowest lying $S^{z}=\frac{1}{2}(0)$ and $S^{z}=\frac{3}{2}(\mathrm{X})$ excitations for the $J_{2}=0.5$ and $\delta=0.05$ Heisenberg chain as a function of the square inverse of the chain length L (up to $\mathrm{L}=25$ ) obtained by ED. The energy reference is the GS for each chain length. Dashed lines represent a $\left(\frac{1}{L^{2}}, \frac{1}{L^{3}}\right)$ fit and the extrapolations to infinite sizes are indicated. DMRG extrapolations are also plotted $(\diamond)$.

The wave functions $\left\langle S_{i}^{z}\right\rangle$ obtained in a DMRG calculation shown in Fig. . 5 clearly support the previous interpretation. The four lowest states $(\mathrm{a}, \mathrm{b}, \mathrm{c}, \mathrm{d})$ show a soliton bound to the weak bond edge, and the soliton moves further away from the impurity when its energy increases. In contrast, in the lowest spin- $\frac{3}{2}$ state (Fig. 国), a $s \bar{s}$ bound pair can be clearly identified in addition to the solitonic GS seen in Fig. (j) (a).

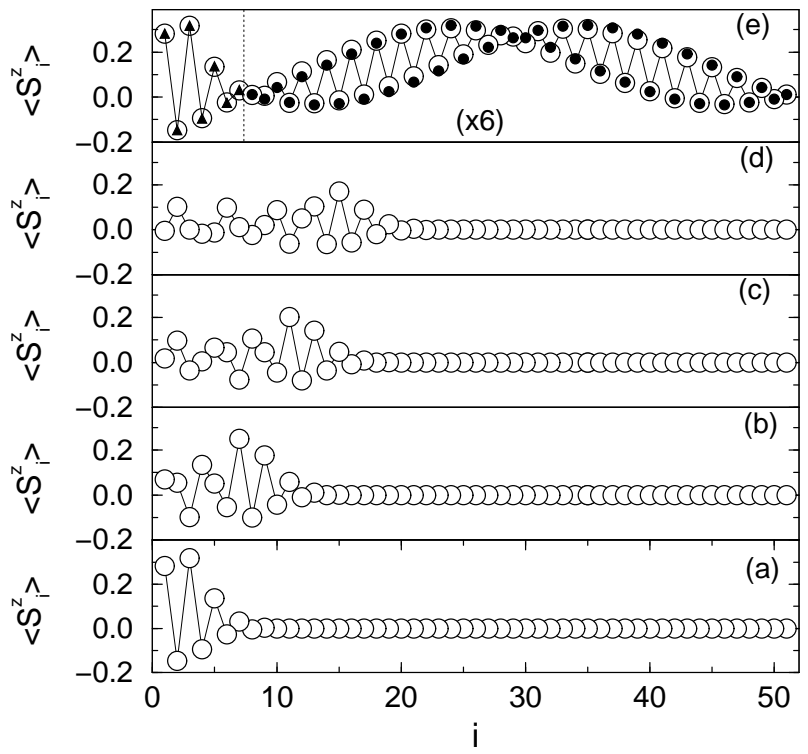

FIG. 5. $\left\langle S_{i}^{z}\right\rangle$ as a function of the site i for the for lowest spin- $\frac{1}{2}(\mathrm{a}, \mathrm{b}, \mathrm{c}, \mathrm{d})$ and the lowest spin- $\frac{3}{2}$ state (e) for $J_{2}=0.5$, $\delta=0.05$ on a 51-site chain using a DMRG algorithm $(\mathrm{m}=128)$. In $(\mathrm{e})$, a magnification by a factor 6 has been realized right to the dashed line. The 7 first sites of the lowest bound state (a) ( $\boldsymbol{\Delta}$ ) and a soliton-antisoliton pair on a 44 site lattice $(\bullet)$ are also shown for comparison. 
As $H_{R}$ is a spin 0 operator, transitions between the $\mathrm{S}=1 / 2$ ground state and higher lying $\mathrm{S}=1 / 2$ bound states can occur and should be identifiable in the Raman spectrum as well-defined peaks at energies $\Delta_{i}^{\mathrm{imp}}$. The Raman spectrum of a 25 site chain with open boundary conditions is shown in Fig. 6 a (plain line) together with the one obtained for a pure 24 site system (dashed line). The low energy structure is clearly due to the presence of the impurities and are signatures of EBS. The high energy part is the bulk contribution and is very similar to the one of the pure system (see section II). The main effect of introducing impurities is then to create additional singlet lines below the singlet-singlet gap $\Delta^{00}$ in the Raman spectrum as has been experimentally observed 8 . The first of the peaks has an energy $\Delta_{1}^{\text {imp }}$ which we interpret as the energy difference between the ground state and the first excited bound state of an odd length chain.

The contribution of the EBS to the total spectrum as compared to the bulk contribution should scale like the impurity concentration i.e. $1 / L$. Hence, in order to correctly identify these states in the relative spectrum the weights are normalized to the impurity concentration. The normalized relative weights of the edge bound states are shown in Fig. 6b for $L$ varying from 11 to 25 sites and $J_{2}=0.5, \delta=0.05$. In this case it is known that four edge bound states exist. Hence, three transition lines should be observable in the Raman spectrum. As shown in Fig. $6 \mathrm{~b}$ the first two transitions between edge bound states have a non-zero weight. The third transition has a very small weight and is almost undetectable.

The presence of the impurity also leads to changes in the BBS resonances. Since the translation invariance is broken, both the $\mathrm{S}=0$ and $\mathrm{S}=1 s \bar{s}$ dispersions are reflected at momenta $q_{n}=n \pi / L$. Consequently, the BBS peaks are split on an energy range corresponding to the respective $s \bar{s}$ bandwidths although they keep almost all their spectral weight. New small satellites should then be observed in Raman experiments forming a quasicontinuum. The possibility of flipping the spin of the impurity bound soliton may lead in rather small systems to a peak at the onset of the $\mathrm{S}=1 s \bar{s}$ quasi-continuum. The weight of this process becomes rapidly negligible for $L>15$ sites (see the weight of the fourth peak in Fig. $6 \mathrm{~b}$ ).

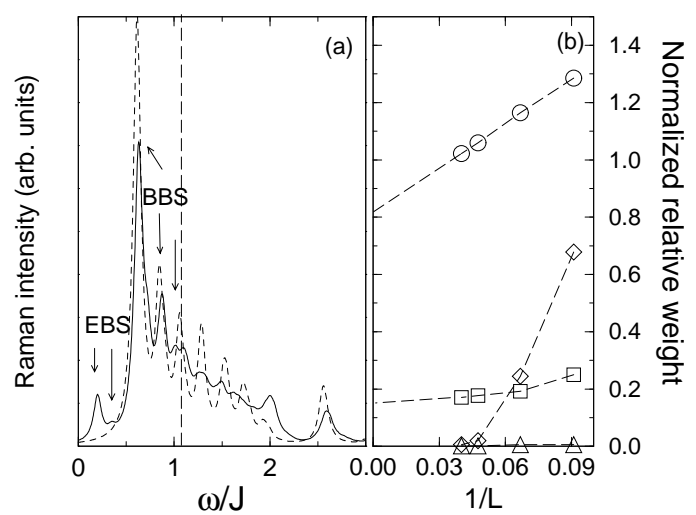

FIG. 6. (a) Raman intensity calculated for a $\mathrm{L}=25$ site open (plain line) and a $\mathrm{L}=24$ site closed chain (dashed line) with $J_{2}=0.5, \delta=0.05$ and a broadening $\varepsilon=0.05 J$. The positions of the first two singlet edge bound state (EBS) $\Delta_{1}^{\text {imp }}$, $\Delta_{2}^{\text {imp }}$ are indicated by arrows, together with the three BBS of Fig. 2. The dashed line shows the onset of the bulk continuum. (b) Relative weight of the four lowest energy peaks in the previous Raman spectrum normalized to the impurity concentration as a function of $1 / L$. The presence of two EBS $(\circ, \square)$ can then be inferred. A third one $(\triangle)$ has an almost zero although non-vanishing weight. The fourth peak $(\diamond)$ corresponds to the first $\mathrm{S}=1 / 2$ state of the quasi-continuum.

For even length open chains, there are two possible scenarios. If the chain begins and ends with a weak bond, the system is equivalent to the addition of two odd chains. Consequently there is a soliton-impurity bound state at each chain end and its edge excitations can be easily inferred from the odd system ones. If both the chain edge bonds are strong no edge bound states exists and the Raman spectrum should be similar to the bulk spectrum for pure systems. Note that this latter case with strong bonds at the edges is favored when lattice relaxation is allowed24.

As a consequence, in agreement with what was proposed in Ref. 25, the previous analysis indicates the presence of low energy peaks below the spin gap in inelastic neutron scattering spectra, due to soliton-impurity bound states.

\section{ROLE OF THE LATTICE RELAXATION}

So far, the underlying lattice was assumed to be frozen in a dimerized configuration. However, recent work suggest that lattice relaxation can be important and lead to qualitatively new physics. Such effects are particularly crucial when inhomogeneities are introduced in the system by, for example, applying a magnetic field26 27 or when doping with impurities24.

In order to go beyond the previous treatment, we shall assume here that the lattice distortion can adjust locally 
to the magnetic modulation of the soliton wavefunction. It has been shownes 24 that, in this case, the soliton acquires both a spin and a lattice component. The description of this magneto-elastic soliton can be realized in term of a simple Hamiltonian,

$$
\begin{aligned}
H & =\sum_{i}\left[J\left(1+\delta_{i}\right) \vec{S}_{i} \cdot \vec{S}_{i+1}+J_{2} \vec{S}_{i} \cdot \vec{S}_{i+2}\right]+\frac{1}{2} K \sum_{i} \delta_{i}^{2} \\
& +\tilde{K}_{\perp} \sum_{i}(-1)^{i} \delta_{i}
\end{aligned}
$$

including an elastic constant $K$ and where the local distortions $\delta_{i}$ are determined self-consistently to minimize the total energy (using e.g. an ED method). The effective interchain coupling $\tilde{K}_{\perp}$ introduced here is, in fact, related to the physical elastic constant $K_{\perp}$ by the self-consistent relation $\check{K}_{\perp}=K_{\perp} \delta$ where $\delta=\left|\left\langle\delta_{i}\right\rangle_{B}\right|$ is the dimerization order parameter in the bulk (pure system). Note that the lattice relaxation which we consider here is purely static (the distortion $\delta_{i}$ varies only in space). A study of quantum lattice fluctuations is beyond the scope of this work (for more details on this issue see e.g. Ref. 29). We then implicitly assume that the time scale of the spin fluctuations is significantly shorter than the one of the lattice (adiabatic approximation). In this case, the ground state $\left|\Psi_{0}\right\rangle$ contains a spin part and a distortion $\left\{\delta_{i}^{0}\right\}$. Since the Raman operator acts on the spin part only and the lattice relaxation time is much larger than the spin relaxation time scale, the eigenstates $\left|\Psi_{n}\right\rangle$ will have the same distortion $\left\{\delta_{i}^{0}\right\}$. Then, once $\left|\Psi_{0}\right\rangle$ is obtained with the selfconsistent method as explained above, the distortion is "frozen" for the computation of the spectra $I_{R}(\omega)$ using Eq. (3).

The interchain coupling $K_{\perp}$ js crucial in order to create a soliton-impurity confinement 24 . The origin of this confinement is clear; in the absence of $K_{\perp}$ the lattice exhibits a solitonic distortion pattern and the point where $\delta_{i}$ vanishes coincides with the maximum of the spin wavefunction $\left\langle S_{i}^{z}\right\rangle$ as seen in Fig. Fa. Hence, in the region between the impurity and the soliton the lattice dimerization in the chain is then out of phase with the order parameter of the bulk, $\left\langle\delta_{i}\right\rangle_{B}=(-1)^{i} \delta$. A finite value of $K_{\perp}$ then leads to an elastic energy cost proportional to the impurity-soliton separation. The new equilibrium position is then obtained when the elastic "force" equilibrates the magnetic pressure that tends to delocalize the soliton away from the wall as shown in Fig. 7 b. When $\tilde{K}_{\perp} \rightarrow \infty$ while the lattice constant $K$ is tuned to maintain a fixed value of the bulk dimerization $\delta\left(K \sim \tilde{K}_{\perp} / \delta \equiv K_{\perp}\right)$, the topological lattice defect is pushed completely towards the impurity end. The lattice becomes then perfectly dimerized and one recovers the previous model.

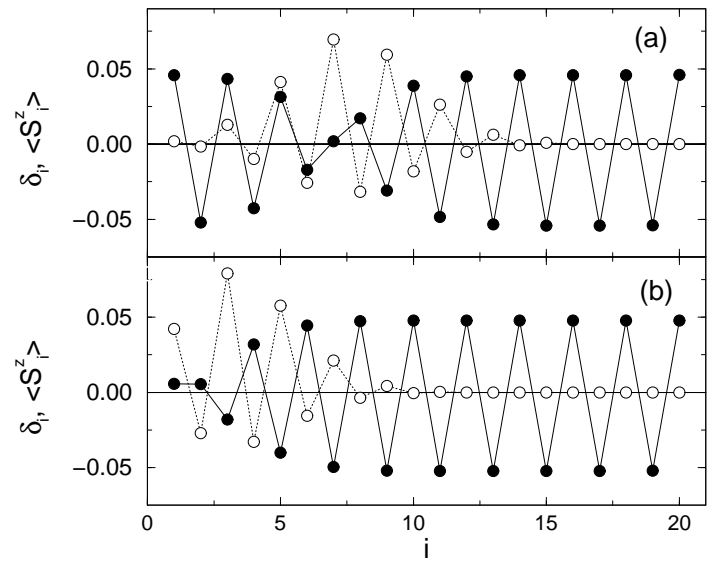

FIG. 7. Results obtained on a $\mathrm{L}=21$ site chain for the spin polarization $\left\langle S_{i}^{z}\right\rangle(\mathrm{o})$ and the lattice distortion $\delta_{i}(\bullet)$ as a function of the site i (the impurity is located on site 0 ) for $J_{2}=0.5$. (a) $K=7.7, K_{\perp} \simeq 0$; (b) $K=11.7, \tilde{K}_{\perp}=0.02$ $\left(K_{\perp} \simeq 4\right)$.

We shall focus here on the modification of the Raman spectrum when $K_{\perp}$ becomes finite i.e. when the lattice can relax as described above. As shown in Figs. 8, the low energy EBS are robust features in the Raman spectrum. Note that we have fixed the bulk value of $\left|\left\langle\delta_{i}\right\rangle_{B}\right|=\delta \simeq$ 0.05 by varying the parameters $K$ and $K_{\perp}$ simultaneously so that a direct comparison with Fig. 6 can be made. The finite size scaling of the weights in Fig. $8 \mathrm{~b}$ unambiguously establishes the existence of well-defined $\delta$-peaks which can be interpreted as soliton bound states.

For decreasing mean-field coupling $\tilde{K}_{\perp}$ important changes in the spectrum take place with respect to the uniformly dimerized chain; (i) the characteristic energies of the EBS resonances decrease and (ii) a transfer of spectral weight occurs from the lowest energy pole to the next one. The three spectra shown in Figs. Ba correspond to $\tilde{K}_{\perp}=0,0.1$ and 0.2 and to (realistic) anisotropy ratios $K_{\perp} / K=0,0.21$ and 0.34 respectively. The energy shift of the two low-energy peaks to lower energy is clearly visible (together with the transfer of weight) when the lattice becomes more and more anisotropic. Indeed, for $\tilde{K}_{\perp}$ decreasing from $\infty$ ("rigidly" dimerized chain) to 0 (isolated $1 \mathrm{D}$ chain), at fixed $\delta=0.05$, the energy of the first resonance decreases from $0.20 J$ to $0.13 J$ while the second peak moves from $0.35 \mathrm{~J}$ to $0.24 \mathrm{~J}$. As the interchain coupling is varied, a large transfer of weight is observed in the spectrum. Indeed, almost all the low energy spectral weight is carried by the first resonance for a large $\tilde{K}_{\perp}$ (with a relative weight in the first peak 0.040 vs. 0.0067 in the second one) whereas this weight is mainly located in the second peak for a vanishing interchain coupling (with a relative weight in the first peak 0.00065 vs. 0.071 in the second one). In this latter case, only one peak, the second one, can be distinguished in the spectrum (see the dotted line in Fig. 8a). 


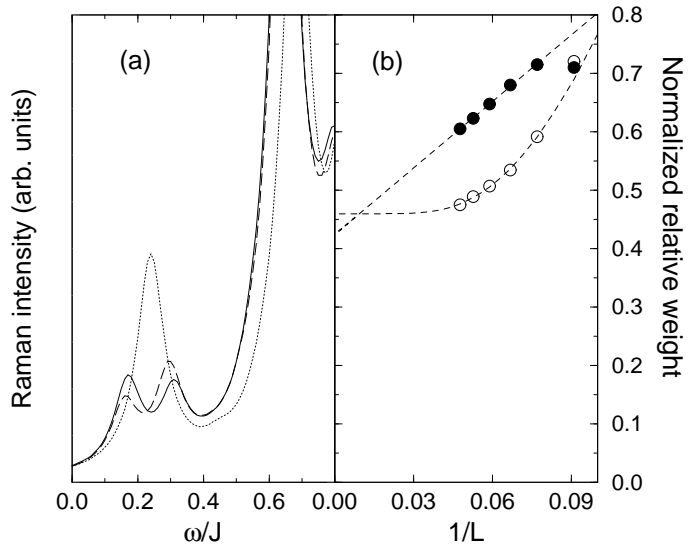

FIG. 8. (a) Raman intensity calculated on a $\mathrm{L}=21$ open chain with an adiabatic lattice for $J_{2}=0.5$ and a broadening $\varepsilon=0.05 J$. Only the low energy region below the BBS $s \bar{s}$ excitation is shown. The dotted, dashed and full lines correspond to $\tilde{K}_{\perp}=0,0.1$ and 0.2 respectively. The values of $K$ ( $=7.7,9.7$ and 11.7 respectively) have been chosen to fix the bulk value $\left|\left\langle\delta_{i}\right\rangle_{B}\right|=\delta \simeq 0.05$. (b) Relative weight of the two lowest energy peaks in one of the previous Raman spectrum $\left(\tilde{K}_{\perp}=0.2, K=11.7\right)$ normalized to the impurity concentration plotted vs $1 / L$. The dashed lines correspond to fits of the data using the form $a+b \frac{\exp (-L / \xi)}{L}$.

\section{RELEVANCE TO CUGEO $_{3}$}

So far all calculations have been performed for parameters close to the MG point in order to minimize finite size effects and clearly identify the bound states. We now discuss the implications of our results for more realistic parameter values relevant to $\mathrm{CuGeO}_{3}$. Parameters such as $J \simeq 160 \mathrm{~K}, J_{2} \simeq 0.36$ and $\delta \simeq 0.014$ have been inferred by ED from high temperature susceptibility-measurements and the zero temperature spin gap value11,2130. Raman spectra obtained by ED would have very large size effects for these parameters, making an interpretation very difficult. Indeed, previous studies for $J_{2}=0$ or $J_{2}=0.24$ hive seen no indication of solitonantisoliton bound state 31 . Hence, we turn to DMRG techniques, first omitting possible lattice relaxation effects. Although the associated spectral weights cannot be obtained, the different gaps can be precisely evaluated using even open chains: $\Delta^{01} / J \simeq 0.1802, \Delta^{00} / J \simeq 0.2529$ and $\Delta^{02} / J \simeq 0.3606$ (see Fig. 9a).

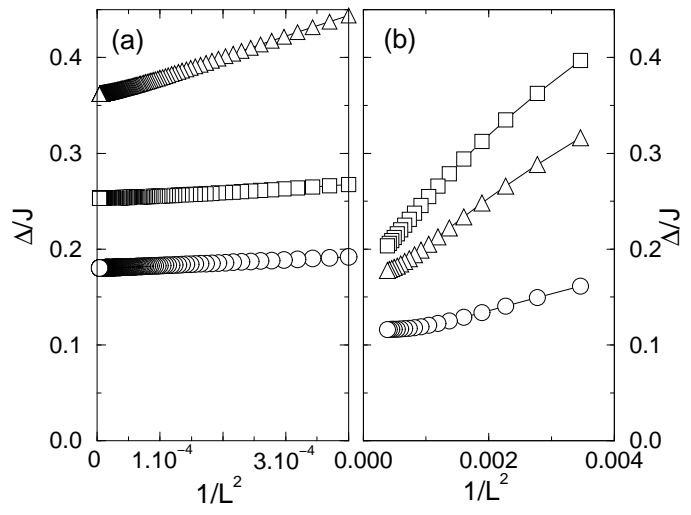

FIG. 9. Variation of (a) the singlet-triplet $\Delta^{01}$ (o), singlet-singlet $\Delta^{00}(\square)$ and singlet-quintuplet gap $2 \Delta^{01}(\triangle)$; (b) the gap between the ground state and the first $\Delta_{1}^{\text {imp }}(\circ)$, second $\Delta_{2}^{\text {imp }}(\triangle)$ and third $\Delta^{01}(\square)$ excited state for $J_{2}=0.36$ and $\delta=0.014$ as a function of the inverse square chain length using a DMRG algorithm for open chains.

Using the same parameter values, it appears that three impurity-soliton bound states (Fig. 10a,b,c) occur for odd open chains. These three bound states have a very extended but still localized wavefunction, signalled by an exponentially decaying part of $\left\langle S_{i}^{z}\right\rangle$ far from the chain end. For instance, the exponential part of the ground state (Fig. 10a) is $\sim \exp (-r / 6.8)$. In contrast, the first $\mathrm{S}=3 / 2$ state shown in Fig. 10d is made of a soliton close to the impurity (identical to the ground state (a) in the thermodynamic limit) plus a soliton-antisoliton pair which extends all over the rest of the chain. Note that the last bound state appears immediately below the first $\mathrm{S}=3 / 2$ state. This is clear for short and intermediate lengths, however, this state could possibly become part of the $s \bar{s}$ quasi-continuum when $L \rightarrow \infty$. Our data seem to indicate that this is not the case.

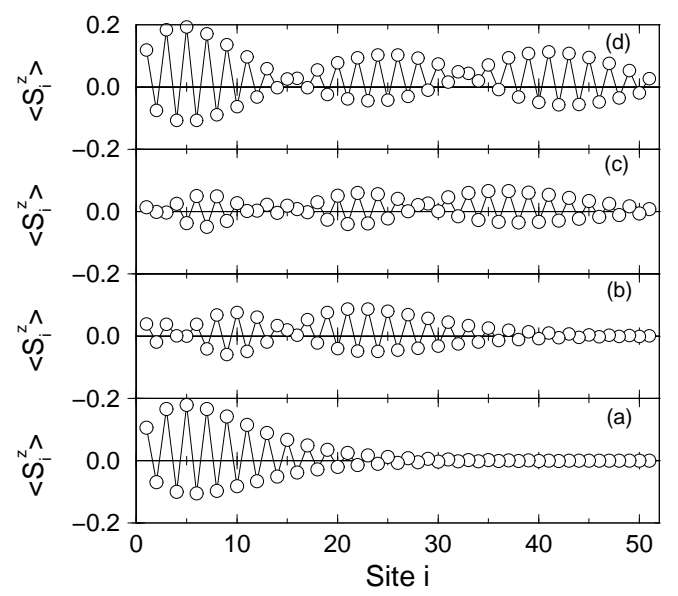

FIG. 10. DMRG results for $\left\langle S_{i}^{z}\right\rangle$ as a function of the site $\mathrm{i}$ for the three lowest $\mathrm{S}=1 / 2$ (impurity-soliton bound states: a, b, c) and the lowest $\mathrm{S}=3 / 2$ state (onset of the quasi-continuum: d) on a 51 site chain beginning with a weak link for $J_{2}=0.36, \delta=0.014$. 
The two gaps associated with these three impuritysoliton bound states have also been evaluated by DMRG (Fig. 9 b): $\Delta_{1}^{\mathrm{imp}} / J \simeq 0.116$, and $\Delta_{2}^{\mathrm{imp}} / J \simeq 0.175$ (just below the quasi-continuum). The energy difference between the ground state and the third excited state, which has a spin $3 / 2$ and is the first state of the quasicontinuum, gives approximately the spin gap $\Delta^{01}$ (see Fig. 9b), as we have explicitly checked. Equivalently, using $J \simeq 160 \mathrm{~K} \simeq 112 \mathrm{~cm}^{-1}$, one obtains: $\Delta^{01} \simeq 20 \mathrm{~cm}^{-1}$, $\Delta^{00} \simeq 28 \mathrm{~cm}^{-1}$, of the same order as the experimental values $\simeq 17 \mathrm{~cm}^{-1}$ and $30 \mathrm{~cm}^{-1}$ respectively. The small deviation between theory and experiments may be attributed to other effects not included in this paper such as magnetic interchain coupling. For the doped systems our results are $\Delta_{1}^{\mathrm{imp}} \simeq 13 \mathrm{~cm}^{-1}$, in surprisingly good agreement with the experimental result $15 \mathrm{~cm}^{-1}$ (Ref. 8). The ratio $\Delta_{1}^{\mathrm{imp}} / \Delta^{01}$ is about 0.6 , which is very similar to what has been found using a particle in a linear potential 8 . However, according to our analysis, another EBS should be observed at $\Delta_{2}^{\text {imp }} \approx 20 \mathrm{~cm}^{-1}$, contrary to the linear potential approach that predicts only ond However, the spectral weight of this state might be very small.

For an anisotropic lattice (see model (4)), another alternative explanation might have to be invoked. Indeed, in that case, significant lattice relaxation would shift the poles to smaller energies. One is then tempted to attribute the experimental resonance at $15 \mathrm{~cm}^{-1}$ to the second theoretical EBS. In this scenario, another EBS is expected with a small weight at energies below $10 \mathrm{~cm}^{-1}$ A direct comparison with the experimental spectrum 8 may corroborate this prediction but the signal noise prevents a definitive conclusion. Furthermore, detection of resonances in Raman scattering experiments at very low energy is difficult due to Brillouin scattering.

In conclusion, we have shown that $s \bar{s}$ bound states should be easily identifiable in experimental Raman spectra on pure systems. Consequently, it is reasonable to interpret the experimentally observed line at $29.7 \mathrm{~cm}^{-1}$ as a $s \bar{s}$ bound state. Doping with chain breaking impurities should give rise to a number of edge bound states with non-negligible Raman weight. At least one such transition has already been experimentally observed 8 in $\mathrm{CuGeO}_{3}$.

We thank IDRIS (Orsay) for allocation of CPU time on the C94 and C98 CRAY supercomputers and rs6000 workstations. We also acknowledge funding from the ECOS-SECyT A97E05 programme. J.R. thanks C.N.R.S. (France) for financial support.

${ }^{1}$ M. Hase, I. Terasaki, and K. Uchinokura, Phys. Rev. Lett. 70, 2651 (1993).
${ }^{2}$ M. Arai, M. Fujita, M. Motokawa, J. Akimitsu and S. M. Bennington, Phys. Rev. Lett. 77, 5579 (1996).

${ }^{3}$ M. Aïn, J. E. Lorenzo, L.-P. Regnault, G. Dhalenne, A. Revcolevschi, B. Hennion and Th. Jolicœur, Phys. Rev. Lett 78, 1560 (1997).

${ }^{4}$ H. Kuroe, T. Sekine, M. Hase, Y. Sasago, K. Uchinokura, H. Kojima, I. Tanaka, and Y. Shibuya, Phys. Rev. B 50, 16468 (1994).

${ }^{5}$ P. H. M. van Loosdrecht, J.-P. Boucher, G. Martinez, G. Dhalenne, and A. Revcolevschi, Phys. Rev. Lett. 76, 311 (1996); P. H. M. van Loosdrecht, J. Zeman, G. Martinez, G. Dhalenne, and A. Revcolevschi, ibid 78, 487 (1997).

${ }^{6}$ V. N. Muthukumar, C. Gros, W. Wenzel, R. Valentì, P. Lemmens, B. Eisener, G. Güntherodt, M. Weiden, C. Geibel and F. Steglich, Phys. Rev. B 54, R9635 (1996).

${ }^{7}$ G. Els, P. H. M. van Loosdrecht, P. Lemmens, H. Vonberg, G. Güntherodt, G. S. Uhrig, O. Fujita, J. Akimitsu, G. Dhalenne and A. Revcolevschi, Phys. Rev. Lett. 79, 5138 (1997).

${ }^{8}$ G. Els, G. S. Uhrig, P. Lemmens, H. Vonberg, P. H. M. van Loosdrecht, G. Güntherodt, O. Fujita, J. Akimitsu, G. Dhalenne and A. Revcolevschi, Europhys. Lett. (in press, 1999).

${ }^{9}$ I. Affleck, Dynamical properties of unconventional magnetic systems (A. T. Skjeltorp and D. Sherrington, Kluwer Academic, Dordrecht, Boston 1998).

${ }^{10}$ E. Sørensen, I. Affleck, D. Augier and D. Poilblanc, Phys. Rev. B 58, R14 701 (1998).

${ }^{11}$ J. Riera and A. Dobry, Phys. Rev. B 51, 16098 (1995).

12 G. Castilla, S. Chakravarty and V. J. Emery, Phys. Rev. Lett. 75, 1823 (1995).

${ }^{13}$ F. D. M. Haldane, Phys. Rev. B 25, 4925 (1982).

${ }^{14}$ K. Okamoto and K. Nomura, Phys. Lett. A 169, 433 (1992).

15 S. Eggert, Phys. Rev. B 54, R9612 (1996).

${ }^{16}$ C. K. Majumdar and D. K. Ghosh, J. Math. Phys. 10, 1399 (1969).

17 B. S. Shastry and B. Sutherland, Phys. Rev. Lett. 47, 964 (1981).

18 G. Bouzerar, A. P. Kampf and G. I. Japaridze, Phys. Rev. B 58, 3117 (1998).

${ }^{19}$ G. S. Uhrig and H. J. Schulz, Phys. Rev. B 54, R9624 (1996).

${ }^{20}$ P. A. Fleury and R. Loudon, Phys. Rev. 166, 514 (1968).

${ }^{21}$ G. Bouzerar and A. P. Kampf and F. Schönfeld, preprint cond-mat/9701176.

22 M. Laukamp, G. B. Martins, C. Gazza, A. L. Malvezzi, E. Dagotto, P. Hansen, A. C. López and J. Riera, Phys. Rev. B 57, 10755 (1998).

${ }^{23}$ G. S. Uhrig, F. Schönfeld, M. Laukamp and E. Dagotto, Eur. Phys. J. B 7, 67 (1999).

${ }^{24}$ P. Hansen, D. Augier, J. Riera and D. Poilblanc, Phys. Rev. B, in press (1999).

${ }^{25}$ G. B. Martins, E. Dagotto and J. Riera, Phys. Rev. B 54, 16032 (1996).

${ }^{26}$ A. Feiguin, J. Riera, A. Dobry and H. Ceccatto, Phys. Rev. B 56, 14607 (1997)

${ }^{27}$ G. S. Uhrig, F. Schönfeld and J. P Boucher, Europhys. Lett. 41, 431 (1998); T. Lorenz et al., Phys. Rev. Lett. 81, 181 (1998). 
${ }^{28}$ H. Nakano and H. Fukuyama, J. Phys. Soc. Jpn. 49, 1679 (1980).

29 D. Augier, D. Poilblanc, E. Sørensen and I. Affleck, Phys. Rev. B 58, 9110 (1998); see also G. Wellein, H. Fehske and A. P. Kampf, Phys. Rev. Lett. 81, 3956 (1998).

${ }^{30}$ K. Fabricius, A. Klümper, U. Löw, B. Büchner, T. Lorenz, G. Dhalenne and A. Revcolevschi, Phys. Rev B 57, 1102 (1998).

${ }^{31}$ D. Augier, D. Poilblanc, S. Haas, E. Delia and E. Dagotto, Phys. Rev. B 56, R5732 (1997). 
Comparison

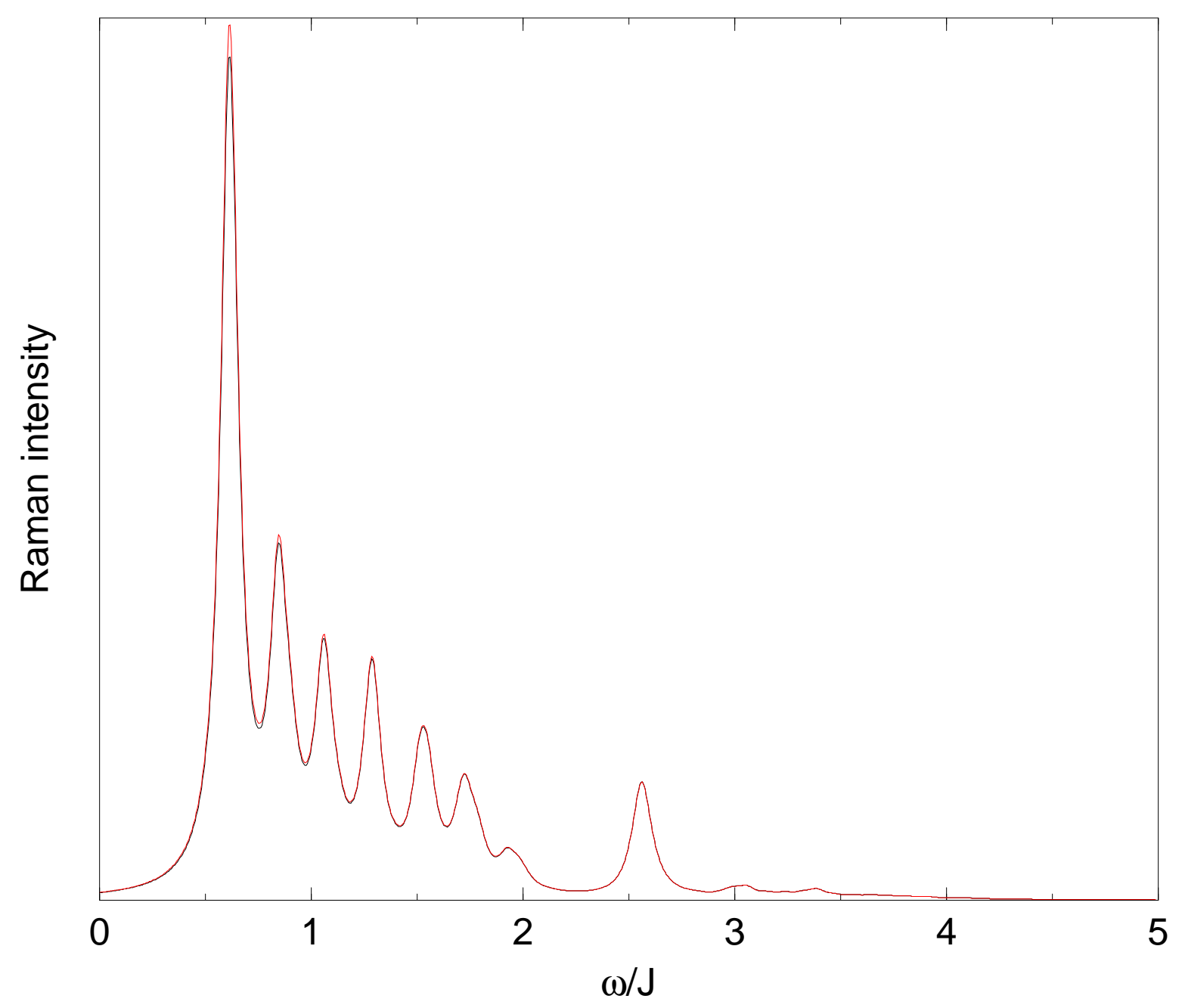

